\title{
Root Microcracks Formation during Root Canal Instrumentation Using Reciprocating and Rotary Files
}

\author{
Rahhali Mohamed ${ }^{1}$, Sakout Majid ${ }^{2}$, Abdallaoui Faiza ${ }^{3}$
}

\begin{abstract}
Aim and objective: The aim of this study was to compare the formation of dentinal cracks with nickel-titanium (NiTi) instruments working in continuous rotation and reciprocating motion.

Materials and methods: One hundred sixty extracted human mandibular first molars were selected for the study. The mesial roots were resected and mounted in resin blocks with simulated periodontal ligaments. Those teeth were randomly assigned to five groups ( $n=32$ teeth/group). The first one was treated with $K$-files and served as control group, and the remaining 128 teeth were divided into 4 groups depending on the root canal preparation technique. Group 2 samples were prepared by sequential ProTaper Universal (PTU), group 3 samples with rotary ProTaper Universal (RPTU), group 4 achieved by the One Shape (OS), and group 5 with the WaveOne (WO) primary files. Roots were then horizontally sectioned at 3,6, and $9 \mathrm{~mm}$ from the apex, and the slices were then observed under a stereomicroscope at $\times 40$ magnification to determine the presence of internal dentinal microcracks. The presence or absence of dentinal defaults was recorded and the statistical analysis was performed by Pearson Chi-square test. The significance level was set at $p<0.05$. The study was made in the oral biology and biotechnology research laboratory of the faculty of medical dentistry of Rabat, Morocco.

Results: No dentinal defect was seen with the stainless steel hand file (group 1). The manual PTU (group 2), the RPTU (group 3), the OS (group 4), and the WO (group 5) in reciprocating motion caused cracks in $15.6 \%, 12.4 \%, 21.9 \%$, and $6.2 \%$ of samples, respectively. The highest percentage of dentinal defect was showed in group 4 but without significant difference with the other group $(p>0.05)$.

Conclusions: Dentinal cracks are produced indifferently of motion kinematics. Within the limits of this study and the current literature, such incidence is less with instruments working in reciprocating motion compared with those working in continuous rotation. Manual and rotary NiTi sequential systems showed fewer microcracks than the single file system working by continuous rotation motion.

Keywords: Continuous rotation, Dentinal cracks, Nickel-titanium rotary files, Reciprocating motion.

The Journal of Contemporary Dental Practice (2021): 10.5005/jp-journals-10024-3055
\end{abstract}

\section{INTRODUCTION}

During the procedure of root canal instrumentation, the canal is shaped by the contact between instruments and dentin walls. This contact may induce defects in the canal walls which are materialized as cracks or microcracks; ${ }^{1}$ these defects can be the point of initiation of root fracture under the effect of occlusal loads. ${ }^{2}$

The goal of endodontic treatment is to ensure a conical root canal shaping respecting the original anatomy of the root canal and compatible with a good dynamic of the irrigation solution to ensure canal disinfection. This result is perpetuated in time with a threedimensional root filling and a coronary functional restoration. ${ }^{3}$

The root canal preparation could be made with manual files or rotary nickel-titanium (NiTi) instruments which could work with different motions. During the canal shaping, dentin is inevitably removed and this may cause dentinal defects. The file's design may influence its behavior on the root canal and consequently the generation of microcracks. Some parameters could be involved like the NiTi core diameter, the cross-sectional shape, the rake angle, and the flute depth. ${ }^{4}$

The ProTaper rotary files (Dentsply Maillefer, Ballaigues, Switzerland) are popular instruments. It is based on a sequence of files in different sizes that are characterized by an increasing taper design, convex triangular cross-section throughout their active portion, and a negative rake angle. ${ }^{5}$ This design allows active cutting motion and a facility to remove more dentin coronally. ${ }^{1}$ This system was reported to generate more dentin damage than other rotary instruments. ${ }^{1,6}$ 1-35aculty of Medical Dentistry, Mohammed V University, Rabat, Morocco

Corresponding Author: Rahhali Mohamed, Faculty of Medical Dentistry, Mohammed V University, Rabat, Morocco, Phone: +212 662222918, e-mail: dr.rahhalisimo@yahoo.fr

How to cite this article: Rahhali M, Sakout M, Abdallaoui F. Root Microcracks Formation during Root Canal Instrumentation Using Reciprocating and Rotary Files. J Contemp Dent Pract 2021;22(3): 259-263.

Source of support: Nil

Conflict of interest: None

In the last decade, we notice the introduction of different singlefile systems in a rotary and reciprocating motion.

The OneShape file (Micro-Mega, Besançon Cedex, France) works in a continuous rotation motion. It has a constant taper of $6 \%$ and an apical diameter of $25 / 100 \mathrm{~mm}$, and it is also two cutting edges in the coronal part, a triangle cutting edge in the apical part, and a cross-section that changes progressively from 3 to 2 cutting edges between the apical and coronal parts; this design offers an optimal cutting action. ${ }^{7}$

The WaveOne (WO) (Dentsply Maillefer) is a NiTi single-file system that works in reciprocating motion; it is manufactured with M-wire NiTi alloy and is more flexible and resistant to cyclic fatigue. Three single-use files are available: small (ISO 21 tip and 6\% taper)

(0) Jaypee Brothers Medical Publishers. 2021 Open Access This article is distributed under the terms of the Creative Commons Attribution 4.0 International License (https://creativecommons.org/licenses/by-nc/4.0/), which permits unrestricted use, distribution, and non-commercial reproduction in any medium, provided you give appropriate credit to the original author(s) and the source, provide a link to the Creative Commons license, and indicate if changes were made. The Creative Commons Public Domain Dedication waiver (http://creativecommons.org/publicdomain/zero/1.0/) applies to the data made available in this article, unless otherwise stated. 
for fine canals, primary (ISO 25 tip and 8\% taper) for the majority of canals, and large (ISO 40 and $8 \%$ taper) when the canals are large. ${ }^{8}$

The use of a single NiTi instrument is interesting since we can reduce the cyclic fatigue and the cross-contamination but it remains more expensive than the conventional multiple files $\mathrm{NiTi}$ rotary systems.

The purpose of the present study was to assess the formation of root cracks in the internal canal walls in multiple levels after root canal instrumentation with different NiTi rotary files and with the stainless steel hand files ( $K$-files $/ H$-files).

\section{Materials and Methods}

One hundred sixty freshly extracted human mandibular first molars were selected for this study and stored in purified filtered water. Teeth with open apices or showing external resorption or curvature upper to $25^{\circ}$ on the mesial root were excluded.

The mesial roots were resected to the long axis of the tooth by using a diamond-coated bur (Diamond Disc, BesQual $0.2 \times 22 \mathrm{~mm}$ Double Edge) mounted on a handpiece (NSK S.Max M65, Japon) with water cooling. The average length of the mesial roots was $17 \mathrm{~mm}$. All roots were inspected with an optical magnifying glass at a magnification $\times 4$ by two examiners to remove any preexisting craze lines or cracks.

The glide path of the mesiobuccal canal was established with \#15 K-File (Dentsply Maillefer, Ballaigues, Switzerland) and the working length of the canals was determined by inserting a size $\# 10 \mathrm{~K}$-file into the root canal terminus and subtracting $1 \mathrm{~mm}$ from this measurement.

Each root was wrapped with a thin layer of hydrophilic vinyl imprint vinyl polysiloxane (OranWash ${ }^{\circledR}$ Zhermack) to simulate the periodontal ligament space and embedded in acrylic resin blocks (Techno Tray-P Protechno) (Fig. 1).

The teeth were randomly divided into five groups $(n=32)$ as follows:

Group 1: Control group: alternance of stainless steel Kerr/Hedström files (HFs) (Dentsply Maillefer) $(n=32)$,

The canals were prepared at the working length until the 25/100 taper. The $K$ files were used by crawling movement and the $H$ files by traction.

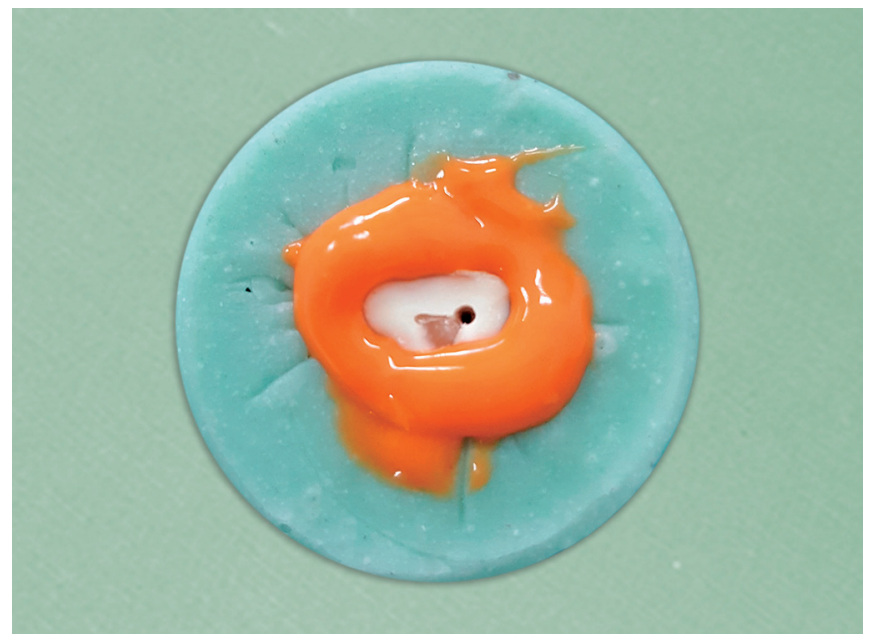

Fig. 1: Root wrapped with a layer of hydrophilic vinyl imprint vinyl polysiloxane and embedded in acrylic resin blocks
After instrumentation, irrigation with $3 \mathrm{~mL}$ of $2.5 \%$ sodium hypochlorite was performed. $K$ and $H$ files were changed as soon as signs of fatigue appear.

Group 2: Manual ProTaper Universal (MPTU) system (Dentsply Maillefer) $(n=32)$,

The sequence was manually used with a clockwise 3/4 turn followed by a $1 / 4$ turn anticlockwise movement followed by a withdrawal.

SX was used to flare the canal orifice then S1, S2, and F1 were used at the working length. The flutes of the instrument were cleaned after each peck.

Group 3: Rotary ProTaper Universal (RPTU) system (Dentsply Maillefer) $(n=32)$,

The sequence of Protaper Universal (PTU) was used to prepare the canals at $300 \mathrm{rpm}$ and $2.4 \mathrm{~N} / \mathrm{cm}$ of torque by using a speedcontrolled motor (X-Smart; Dentsply Tulsa Dental, Tulsa, OK).

The Shaping File $X$ was used in coronal enlargement, and S1, $\mathrm{S} 2$ with a brushing motion away from the root concavities. Finally, F1 file was used until the working length was reached and which correspond to apical size 20 . The flutes of instruments were cleaned after each peck.

Group 4: One Shape (OS) system (Micro-Mega) ( $n=32$ ),

Canal preparation was performed with OS rotary file (MicroMega, Besancon Cedex, France) by using an X-Smart motor (Dentsply Tulsa Dental, Tulsa, OK) at a constant speed of $400 \mathrm{rpm}$ and $2.4 \mathrm{~N} / \mathrm{cm}$ of torque. The OS was used at $2 / 3$ of the working length, then at the WL-3 mm and finally at the WL by cleaning the flutes of the instrument after each peck.

\section{Group 5: WO system primary (Dentsply Maillefer) $(n=32)$}

WO taper $8 \%$ and size $25 / 100$ reciprocating file was introduced into the root canals in slow in and out pecking motions with about an amplitude of $3 \mathrm{~mm}$ according to the manufacturer's instructions. The WO instrument was mounted on an endodontic motor (ATS Dental, Italie) by a reciprocity motion. The instrument was removed after two or three pecking motions or when we feel a blockage.

The MPTU, RPTU, and OS were changed after shaping five canals. The WO was changed after three canals.

Irrigation was performed with $2 \mathrm{~mL}$ of $2.5 \%$ sodium hypochlorite between each instrument during the preparations of root canals.

All groups

When the preparation was achieved, irrigation was applied by $2 \mathrm{ml}$ of ethylenediaminetetraacetic acid $15 \%$ (EDTA ${ }^{\oplus}$ PrevestDenPro ${ }^{\oplus}$ ) activated manually with an F1 gutta cone for 2 minutes.

Final irrigation with $5 \mathrm{~mL}$ of $\mathrm{NaOCl} 2.5 \%$ was realized and activated manually with an F1 gutta cone for 2 minutes.

One operator performed all root canal preparations and the teeth were stored in purified filtered water throughout the experiment.

Sectioning and microscopic examination

After preparation, all the roots were sectioned horizontally at $3 \mathrm{~mm}$ (apical third), $9 \mathrm{~mm}$ (middle third), and $15 \mathrm{~mm}$ (cervical third) from the apex with a diamond-coated bur (Diamond Disc, BesQual $0.2 \times 22 \mathrm{~mm}$ double edge) mounted on handpiece (NSK S.Max M65, Japon) with water cooling.

Digital images of each section were captured at $\times 40$ magnification using a digital camera (TOUPCAM ${ }^{\mathrm{TM}}$ U3CMOS) attached to a stereomicroscope (Optika inversé IM-3, Italy). 
Each section was checked by one operator for the presence of internal dentinal defects (microcracks). "No defect" was defined as root dentin devoid of any craze lines or microcracks at the internal surface of the root canal wall. "Defect" was defined as any lines, microcracks, or fractures present in root dentin. A total of 96 sections were examined in each group.

The study was made in the oral biology and biotechnology research laboratory (LRBOB) of the faculty of medical dentistry of Rabat, Morocco.

\section{Results}

The results were expressed as the number and percentage of cracked roots in each group. The $\mathrm{Chi}$-square and Fisher exact tests were used for statistical analysis of differences between groups. The level of significance was set at $p<0.05$. All statistical analyses were performed using SPSS software (SPSS Inc, Chicago, IL).

The HF group did not present any defects. We noticed defects in all the other groups (Fig. 2) and one single case of complete fracture was observed in the RPTU group (Fig. 3).

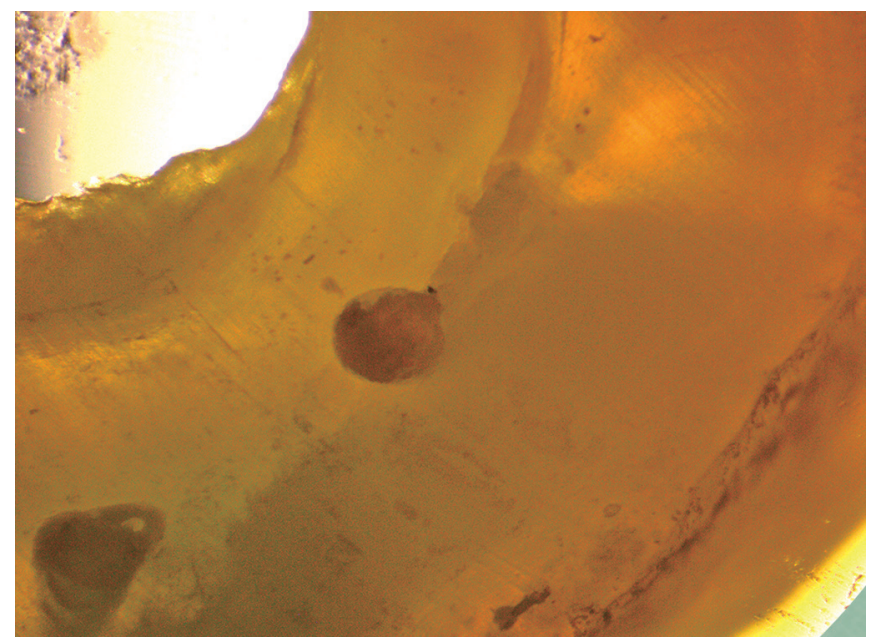

Fig. 2: Section of the middle third showing microcracks in the OneShape group

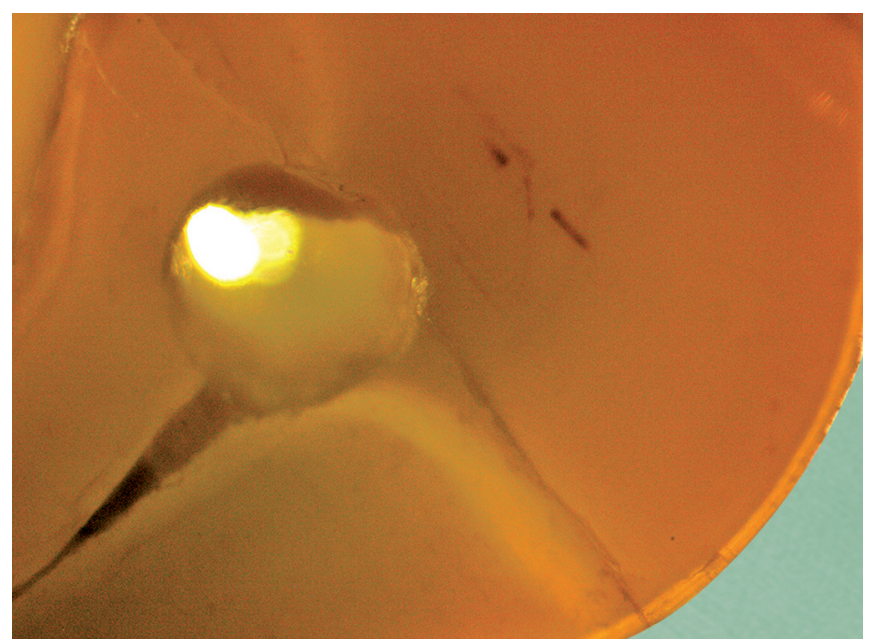

Fig. 3: Section of cervical third showing a complete fracture in the RPTU group
Table 1: The percentage and number of dentinal defects after canal preparation with RPTU, OS, and WO

\begin{tabular}{lllll}
\hline & RPTU & OS & WO & Pvalue \\
\hline Cervical third $(15 \mathrm{~mm})$ & $2(6.3 \%)$ & $1(3.1 \%)$ & $1(3.1 \%)$ & 0.77 \\
Middle third $(9 \mathrm{~mm})$ & $0(0 \%)$ & $3(9.4 \%)$ & $1(3.1 \%)$ & 0.16 \\
Apical third $(3 \mathrm{~mm})$ & $2(6.3 \%)$ & $3(9.4 \%)$ & $0(0 \%)$ & 0.22 \\
\hline
\end{tabular}

Table 2: The percentage and number of dentinal defects after canal preparation with MPTU and RPTU

\begin{tabular}{llll}
\hline & MPTU & RPTU & Pvalue \\
\hline Cervical third $(15 \mathrm{~mm})$ & $2(6.3 \%)$ & $2(6.3 \%)$ & 0.69 \\
Middle third $(9 \mathrm{~mm})$ & $0(0 \%)$ & $0(0 \%)$ & - \\
Apical third $(3 \mathrm{~mm})$ & $3(9.4 \%)$ & $2(6.3 \%)$ & 0.5 \\
\hline
\end{tabular}

The number and percentage of dentinal defects in each group were shown in Tables 1 and 2. The highest percentage of dentinal defect was showed in the group.

\section{Discussion}

The vertical root fracture is not an instantaneous phenomenon but it is a consequence of the propagation of microcracks and defects regarding the root dentine..$^{9,10}$

The aim of this study was to investigate whether there was a statistically significant difference in the prevalence of dentinal defects between:

- Group 1: Control group: alternance of Kerr/Hedström files on stainless steel (Dentsply Maillefer),

- Group 2: MPTU system (Dentsply Maillefer),

- Group 3: RPTU system (Dentsply Maillefer),

- Group 4: OS system (Micro-Mega),

- Group 5: WO system primary (Dentsply Maillefer),

Only the mesiobuccal canal of the mesial roots of the mandibular molars was prepared in order to keep the mesiolingual canal as a control. The dentinal defects concerning the external surface of the root have been removed since they may be generated by the method of section used (diamond disc), so only the defects interesting internal dentinal surfaces have been retained. The placement of a thin layer of hydrophilic vinyl imprint vinyl polysiloxane is important to simulate the periodontal ligament function since it absorbs the stress generated on the tooth by the root canal preparation.

Through the description of the results obtained, it is observed that the manual instrumentation of $K$ and $H$ files does not cause dentinal defects; this is in agreement with the studies carried out previously and which have reported a complete absence of microcracks with manual instrumentation. ${ }^{10,11}$

In the other groups, manual, rotary, and reciprocity NiTi files caused dentinal defects and both the dynamics of reciprocity and continuous rotation would generate dentinal defects. ${ }^{12}$

The comparison of the rotary system shows that there is no statistically significant difference between the reciprocating system (group 5) and both groups 3 and 4 which work in continuous rotation $(p>0.05)$. Despite this, we notice a fewer percentage of internal dentinal defects in the WO group (6.2\%). This could be due to the low torsional and bending forces generated by the reciprocal 
movement compared to the continuous rotational movement. ${ }^{13,14}$ Some authors have suggested that reducing the angle of reciprocity would increase the risk to generate defects. ${ }^{15}$

MPTU, RPTU, and OS systems operating in continuous rotation showed the most dentinal defects without statistically significant difference between the three groups $(p>0.05)$. The greater prevalence of microcracks concerns the single-file group represented by the OneShape (21.9\%).

This could be explained by the file design and the importance of torsional forces developed between the instrumental surface and the canal walls. The speed setting could also be involved since the OS works with higher speed than the RPTU.

The contact area between the OS file and the canal walls is more important than the sequential systems which limit the contact area for each instrument in the sequence to the portion to be prepared, this is why most studies show a high prevalence of dentinal defects with monoinstrumental systems in continuous rotation compared to systems using a sequence file in continuous rotation like the study made by Harandi et al. ${ }^{16}$

The taper increased of files in dynamic systems offers canal preparations more taper, but it would contribute to the weakening of the root structure by a significant reduction in the thickness of the parietal dentine. ${ }^{17}$

We did not show a statistically significant difference between the group of MPTU and the RPTU while the number of files, the design, and taper are the same. The percentage of cracks in the group of MPTU is higher than the percentage checked with the RPTU. This could be from the difficulty to standardize the downward force applied by the operator in the manual group.

The lowest percentage of microcracks was observed in the WO group, the reciprocating motion reduces torsional and flexural stresses ${ }^{13}$ and minimizes canal transportation. ${ }^{18}$

The primary WO uses in this experiment have an apical size of \#25.08 and caused fewer microcracks than the MPTU group, the RPTU group, and the OS group. We could explain this difference by the cross-sectional design of the WO and also by the dynamic of reciprocation. This motion could prevent continuous rotational force and constant torque applied by the NiTi rotary file on the root canal walls and generates as a result fewer microcracks than the continuous rotational motion. ${ }^{19,20}$

According to the study of Bertan et al., the thermomechanical treatment of the NiTi files seems does not have an effect on the creation of microcracks. All the thermo-treated NiTi files used in this study produced dentinal defects. ${ }^{21}$

\section{Conclusion and Clinical Significance}

Under the experimental conditions and the limitations of this study, we can conclude that the stainless steel hand $K / H$ files did not produce defects on the root canals at any level. But both the rotary and reciprocating NiTi files caused the formation of microcracks.

The RPTU and the OS working by continuous rotation motion produced more microcracks than the WO working by reciprocity. The manual and rotary NiTi multifile systems showed fewer microcracks than the single file system working by continuous rotation motion.

We can deduce that a reciprocating system allows more security against microcracks formation compared to the continuous rotation motion and in this dynamic; multifile systems are suitable for a single file system.

\section{Clinical Significance}

We can within the limits of this study, draw some clinical significations:

- The stainless steel hand file does not cause any dentinal damage.

- Both the rotational and reciprocating NiTi files caused dentinal microcracks.

- More microcracks were seen with the system working by continuous rotation motion in comparison with reciprocating NiTi files.

- In continuous rotation dynamic, the NiTi sequential systems showed fewer microcracks than the single file system.

\section{ACKnOWLedgments}

The authors deny any conflicts of interest related to this study. The study was realized in the LRBOB attached to the faculty of dentistry and the University of Mohammed V of Rabat. The biostatistic analysis was realized by the laboratory of epidemiology and clinical research of the University of Mohammed V of Rabat.

\section{References}

1. Bier $\mathrm{C}$, Shemesh $\mathrm{H}$, Tanomaru-Filho $\mathrm{M}$, et al. The ability of different nickeltitanium rotary instruments to induce dentinal damage during canal preparation. J Endod 2009;35:236-238. DOI: 10.1016/j. joen.2008.10.021.

2. Tsesis I, Rosen E, Tamse A, et al. Diagnosis of vertical root fractures in endodontically treated teeth based on clinical and radiographic indices: a systematic review. J Endod 2010;36(9):1455-1458. DOI: 10.1016/j.joen.2010.05.003.

3. Hülsmann M, Peters O, Dummer P. Mechanical preparation of root canals: shaping goals, techniques and means. Endod Topics 2005;10:30-76. DOI: 10.1111/j.1601-1546.2005.00152.x.

4. Kim H, Lee M, Yum J, et al. Potential relationship between design of nickel-titanium rotary instruments and vertical root fracture. J Endod 2010;36:1195-1199. DOI: 10.1016/j.joen.2010.02.010.

5. Bergmans L, Van Cleynenbreugel J, Beullens M, et al. Smooth flexible versus active tapered shaft design using NiTi rotary instruments. Int Endod J 2002;35:820-828. DOI: 10.1046/j.1365-2591.2002.00574.x.

6. Hin E, Wu M, Wesselink P, et al. Effects of self-adjusting file, Mtwo, and ProTaper on the root canal wall. J Endod 2013;39:262-264. DOI: 10.1016/j.joen.2012.10.020.

7. Liu R, Hou B, Wesselink P, et al. The incidence of root microcracks caused by 3 different single-file systems versus the ProTaper system. J Endod 2013;39(8):1054-1056. DOI: 10.1016/j.joen.2013.04.013.

8. Burklein S, Hinschitza K, Dammaschke T, et al. Shaping ability and cleaning effectiveness of two single file systems in severely curved root canals of extracted teeth: reciproc and wave one versus Mtwo and ProTaper. Int Endod J 2012;45:449-461. DOI: 10.1111/j.13652591.2011.01996.x.

9. Bürklein $S$, Tsotsis $P$, Schafer E. Incidence of dentinal defects after root canal preparation: reciprocating versus rotary instrumentation. J Endod 2013;39(4):501-504. DOI: 10.1016/j.joen.2012.11.045.

10. Çiçek E, Koçak M, Saglam B, et al. Evaluation of microcrack formation in root canals after instrumentation with different NiTi rotary file systems: a scanning electron microscopy study. Scanning 2015;37(1):49-53. DOI: 10.1002/sca.21178.

11. Ustun Y, Aslan T, Sagsen B, et al. The effects of different nickeltitanium instruments on dentinal microcrack formations during root canal preparation. Eur J Dent 2015;9(1):41-46. DOI: 10.4103/13057456.149638.

12. Pop I, Manoharan A, Zanini F, et al. Synchrotron light-based $\mu C T$ to analyse the presence of dentinal microcracks post-rotary and reciprocating NiTi instrumentation. Clin Oral Investig 2015;19(1):11-16. DOI: 10.1007/s00784-014-1206-5. 
13. Blum J, Machtou P, Esber S, et al. Analysis of forces developed during root canal preparation with the balanced force technique. Int Endod J 1997;30:386-396.

14. Kansal R, Raiput A, Talwar S, et al. Assessment of dentinal damage during canal preparation using reciprocating and rotary files. J Endod 2014;40(9):1443-1446. DOI: 10.1016/j.joen.2014.02.015.

15. Karataş $E$, Arsian $H$, Alsancak $M$, et al. Incidence of dentinal cracks after root canal preparation with twisted file adaptive instruments using different kinematics. J Endod 2015;41:1130-1133.

16. Harandi A, Mirzaeerad S, Mehrabani M, et al. Incidence of dentinal crack after root canal reparation by ProTaper universal, neolix and safesider systems. Iran Endod J 2017;12(4):432-438. DOI: 10.22037/ iej.v12i4.17597.

17. Priya N, Chandrasekhar V, Anita S, et al. "Dentinal microcracks after root canal preparation" a comparative evaluation with hand, rotary and reciprocating instrumentation. J Clin Diagn Res 2014;8(12):ZC70ZC72. DOI: 10.7860/JCDR/2014/11437.5349.

18. Franco V, Fabiani C, Taschieri S, et al. Investigation on the shaping ability of nickeltitanium files when used with a reciprocating motion. J Endod Topics 2011;37:1398-1401.

19. Berutti E, Paolino D, Chiandussi G, et al. Root canal anatomy preservation of WaveOne reciprocating files with or without glide path. J Endod 2012;38:101-104. DOI: 10.1016/j.joen.2011.09.030.

20. Berutti E, Chiandussi G, Paolino D, et al. Canal shaping with WaveOne primary reciprocating files and ProTaper system: a comparative study. J Endod 2012;38:505-509. DOI: 10.1016/j.joen.2011.12.040.

21. Bertan $\mathrm{K}$, Burak S, Tugrul A. Evaluation of dentinal defects during root canal preparation using thermomechanically processed nickel-titanium files. Eur J Dent 2017;11:157-161. DOI: 10.4103/ejd. ejd_254_16. 